\title{
Unveiling the molecular mechanisms of drought stress tolerance in rice (Oryza sativa L.) using computational approaches
}

\author{
ZAHRA ZiNATI *, VAHID BARATI \\ Department of Agroecology, College of Agriculture and Natural Resources of Darab, Shiraz University, Iran
}

\begin{abstract}
The devastating impact of drought on rice yield as a serious problem has been demanding an enormous effort to develop drought tolerant rice varieties. To start with, one of the major challenges in this regard is identifying the genes and pathways associated with drought tolerance for effectively governing the factor in rice. In the present study, a variety of computational biology techniques including Gene Ontology (GO) enrichment analysis, Kyoto Encyclopedia of Genes and Genomes (KEGG) pathway analysis, and gene network analysis were applied on putative candidate drought-tolerant genes (previously identified via microarray analysis) to decipher the biological processes and pathways by which tolerant genotype copes with drought stress. In terms of biological process, GO enrichment analysis revealed the significance of developmental process, signaling, reproduction, cell death, small molecule metabolic process, oxidation reduction, secondary metabolic process, and response to stimulus. In addition, the majority of uniquely upregulated genes were strongly associated with "response to stimulus". Significantly, from the point of view of molecular function ontology, the uniquely upregulated genes were enriched in serine-type endopeptidase inhibitor activity ( 5 genes). Utilizing KEGG pathway enrichment analysis underscored glutathione metabolism as a critical pathway in tolerant genotype imposed on drought stress. Also, network analysis highlighted UvrD helicase as the central gene. According to the results, the tolerance of tolerant genotype seems to be related to high antioxidant and DNA repair systems efficiencies. This study provides a potent approach for uncovering the crucial genes and pathways that may be contributing to drought tolerance.
\end{abstract}

Key words rice, Gene Ontology enrichment, network analysis, pathway analysis, drought tolerance

\section{Introduction}

Drought stress, one of the most detrimental abiotic stresses limiting rice productivity in $20 \%$ of the total rice growing area in Asia (Pandey, 2007), can considerably lead to even a $60 \%$ loss of the yield (Sarris, 2004; Farooq et al., 2009). Hence, enhancing drought tolerance is of high-priority in rice breeding. Given the most detrimental challenges in this regard, one can highlight the identification of stress responsive genes and pathways involved in regulation of the sophisticated trait of drought tolerance in rice. One strategy to accomplish this would be investigating the underpinning biological processes and pathways of drought tolerance to identify pivotal genes. However, the knowledge on significant biological processes and pathways governing drought tolerance is still limited.

It is well established that drought tolerance is a sophisticated trait consisting of a myriad of morphological and metabolic adaptive pathways such as increased root growth, stomatal closure, leaf rolling, increased ABA production (Price et al., 2002). Thereby, introducing a single gene has not been very successful in dramatically improving the tolerance to drought (Bohnert et al., 2001). In an attempt towards accomplishing pre-set targets, an ongoing shift of focus and transformation in a number of genes (single to multigenes) has been spotted and acknowledged by genetic engineering researchers (Halpin, 2005).

\footnotetext{
* Corresponding author: Department of Agroecology, College of Agriculture and Natural Resources of Darab, Shiraz University, Iran; e-mail: zahrazinati@shirazu.ac.ir
} 
Plant's responses to environmental stresses can be understood thoroughly thanks to genome-wide transcriptional analysis. A comparative transcriptome analysis of rice genotypes showing contrasting responses to drought stress is proved useful in understanding the significant biological processes and pathways which may lead to a tolerant genotype success in coping with drought stress (Lenka et al., 2011; Borah et al., 2017). Furthermore, recent advances in GO enrichment, pathway enrichment and network analysis provide a new insight to investigation of the molecular and biological aftereffects of the transcriptional alterations that occur in response to stresses (Ahn et al., 2017; Wei et al., 2018). Not to mention that the significantly enriched GO terms and pathways can help to further broaden our knowledge of the specific role played by genes in conferring drought tolerance. If it was not for the network analysis which cumulates available information within the networks frame, such important interactions including genes or proteins along with their correlation with each other would have been the matters of obscurity and remained unacknowledged (Cruts et al., 2012).

Moreover, put in a larger scale, determining of key GO terms, pathways and gene network analysis provides an efficient and reliable approach for identifying crucial genes and pathways for effective governing of drought tolerance in rice. Zinati (2017) analyzed the data series (GSE21651) of public rice microarray data (from NCBI gene expression omnibus) produced under drought-stress conditions and identified 308 probe sets upregulated (fold change equal to or greater than 3 ) in a drought tolerant genotype and downregulated (fold change less than 1) in a drought sensitive genotype upon exposure to drought stress. The study was based upon a hypothesis that genes, which are uniquely upregulated in a tolerant genotype, would provide the strongest candidates for drought tolerance in rice.

The current study was designed to include the uniquely upregulated genes in a tolerant rice genotype in the context of GO enrichment analysis, and Kyoto Encyclopedia of Genes and Genomes (KEGG) pathway enrichment analysis. Furthermore, the genes enriched in important biological processes, molecular functions, cellular components and pathways were discussed. In addition, the protein-protein interaction networks of the uniquely upregulated genes were analyzed.

\section{Material and methods}

\section{GO enrichment analysis}

In the current study, the identified 308 in Zinati (2018) probe sets were taken for further analysis. To make some meaningful biological inference from 308 probe sets, GO enrichment analysis was carried out using the AgriGO web-based tool (Du et al., 2010), available from http://bioinfo.cau.edu.cn/agriGO/index.php. The Singular Enrichment Analysis (SEA) was done setting "Rice TIGR gene model" as reference, "Yekutieli (FDR under dependency)" as multi-test adjustment method, "Fisher" as statistical test method, 0.05 as p-value cut-off and " 5 " as minimum number of mapping entries. Affymetrix probe sets were converted to MSU gene locus using DOG-GSEA (Gene Set Enrichment Analysis toolkit with Down-weighting of Overlapping Gene) (http: //doggsea.ncpgr.cn/) and used as queries. After that, the genes were annotated and defined according to the GO terms under the three main categories, namely, biological process, molecular function and cellular component. Further analysis was focused on the set of genes under important biological processes. Similarly, GO enrichment analysis was carried out for genes forming the network.

\section{KEGG pathway enrichment analysis}

To find critical pathways closely related to drought tolerance, KEGG enrichment analysis was carried out using Comprehensive Annotation of Rice Multi-Omics (CARMO). According to Wang and coworkers (2015), CARMO (http://bioinfo.sibs.ac.cn/carmo) is a comprehensive annotation platform for functional exploration of rice multi-omics data with FDR threshold $\leq 0.01$. GO annotation was also executed via CARMO to characterize genes enriched in the key pathway.

\section{Gene network analysis}

Network analysis paves the way for identification of central genes (genes with higher number of connections along with those connecting different networks). Gene interaction network was generated for uniquely upregulated genes using the "The Rice Interactions Viewer" web based tool version Interactome 2.0 (http://bar. utoronto.ca/interactions/cgi-bin/rice_interactions _viewer.cgi) developed by the Bio-Analytic Resource- the 
BAR setting "Filter output to remove duplicate interactions" and "Extend with interactions from BIND/Interactome 2.0" (http://bar.utoronto.ca/welcome.htm). The Rice Interactions Viewer queries a database of 37472 predicted and 430 verified rice interacting proteins (http//bar.utoronto.ca/interactions/cgi-bin/ rice_interactions_viewer.cgi). The interactions predicted are based on experimentally determined orthologous interactions between rice and other eukaryotes. Each interaction has been assigned a confidence level (CV) as an internal quality control (Ho et al., 2012). Moreover, STRING database (http:// string-db.org/) (Szklarczyk et al., 2017) was used to predict protein-protein interactions of identified critical genes.

\section{Identification of critical genes}

All relations in the network were transferred to Cytoscape software, version 2.5.0 (Shannon et al., 2003). Then, Cyto-Hubba, as a hub finder/analyzer software, was used to find critical proteins/hubs by several topological algorithms, including Betweenness centrality, Bottleneck (BN), Closeness centrality, Clustering coefficient, Degree, Density of Maximum Neighborhood Component (DMNC), EcCentricity, Edge Percolated Component (EPC), Maximal Clique Centrality (MCC), Maximum Neighborhood Component (MNC), Radiality centrality, Stress centrality (Chin, et al., 2014). Finally, based on overall results of applying different algorithms some genes were announced as the critical genes.

\section{Promoter analysis}

In order to recognize the promoter regions of genes critical for drought tolerance, nucleotide sequences of $1.5 \mathrm{Kbp}$ upstream from the translation start sites were identified using Phytozome v12.1 (Goodstein et al., 2012). Then, the promoter regions were scanned for the presence of Cis-Acting Regulatory Elements using PlantCARE, a web-based database of plant promoters and their Cis-Acting Regulatory Elements (http://bio informatics.psb.ugent.be/webtools/plantcare/html/) (Lescot et al., 2002).

\section{Expression analysis of critical genes in rice various organs and developmental stages}

In silico expression profiles of the genes critical for drought tolerance were evaluated at rice developmental stages and anatomical compartments by retrieving the values of expression from affymetrix array database of Genevestigator (https://www.genevestigator.com/gv/ (Zimmermann et al. 2004). Affymetrix Rice Genome Array was selected for the microarray data analysis.

\section{Results}

\section{GO enrichment analysis}

GO enrichment analysis was utilized to gain an insight into the meanings of underlined biological, molecular and cellular components from uniquely upregulated probe sets in a tolerant rice genotype. It was observed that 29 out of 308 Affymetrix probe sets did not match the specific MSU gene loci. The remaining $279 \mathrm{MSU}$ gene loci were used as queries for the subsequent bioinformatics analysis. Afterwards, the genes were annotated according to the GO terms under the 3 main categories, namely biological process, molecular function and cellular component. Based on GO enrichment analysis the biological process such as developmental process (GO:0032502) (6 genes), signaling (GO:0023052) (14 genes), reproduction (GO:0000003) (5 genes), cell death (GO:0008219) (12 genes), cellular amino acid and derivative metabolic process (GO:0006519) (12 genes), oxidation reduction (GO:0055114) (5 genes), secondary metabolic process (GO:0019748) (11 genes), response to stimulus (GO:0050896) (50 genes) (Fig. 1) were significantly enriched by identified probe sets. In terms of molecular functions GO enrichment analysis yielded genes enriched in serine-type endopeptidase inhibitor activity (GO:0004867) (5 genes) among the uniquely upregulated ones (Fig. 2). Furthermore, to determine the location of the uniquely upregulated genes, cellular component enrichment analysis traced them mainly in extracellular regions, intracellular membrane-bounded organelles such as plastids, endoplasmic reticulum, cytoplasm, and mitochondria, as well plasma membrane, and cell walls (Fig. 3 ).

\section{KEGG pathway enrichment analysis}

KEGG pathway enrichment analysis was performed on uniquely upregulated genes in a tolerant genotype to characterize the key pathways which may lead to a tolerant genotype success in coping with drought stress. It unraveled uniquely upregulated genes significantly enriched in glutathione metabolism pathway (osa00480) (Table 1). To be more specific, 5 genes among uniquely 


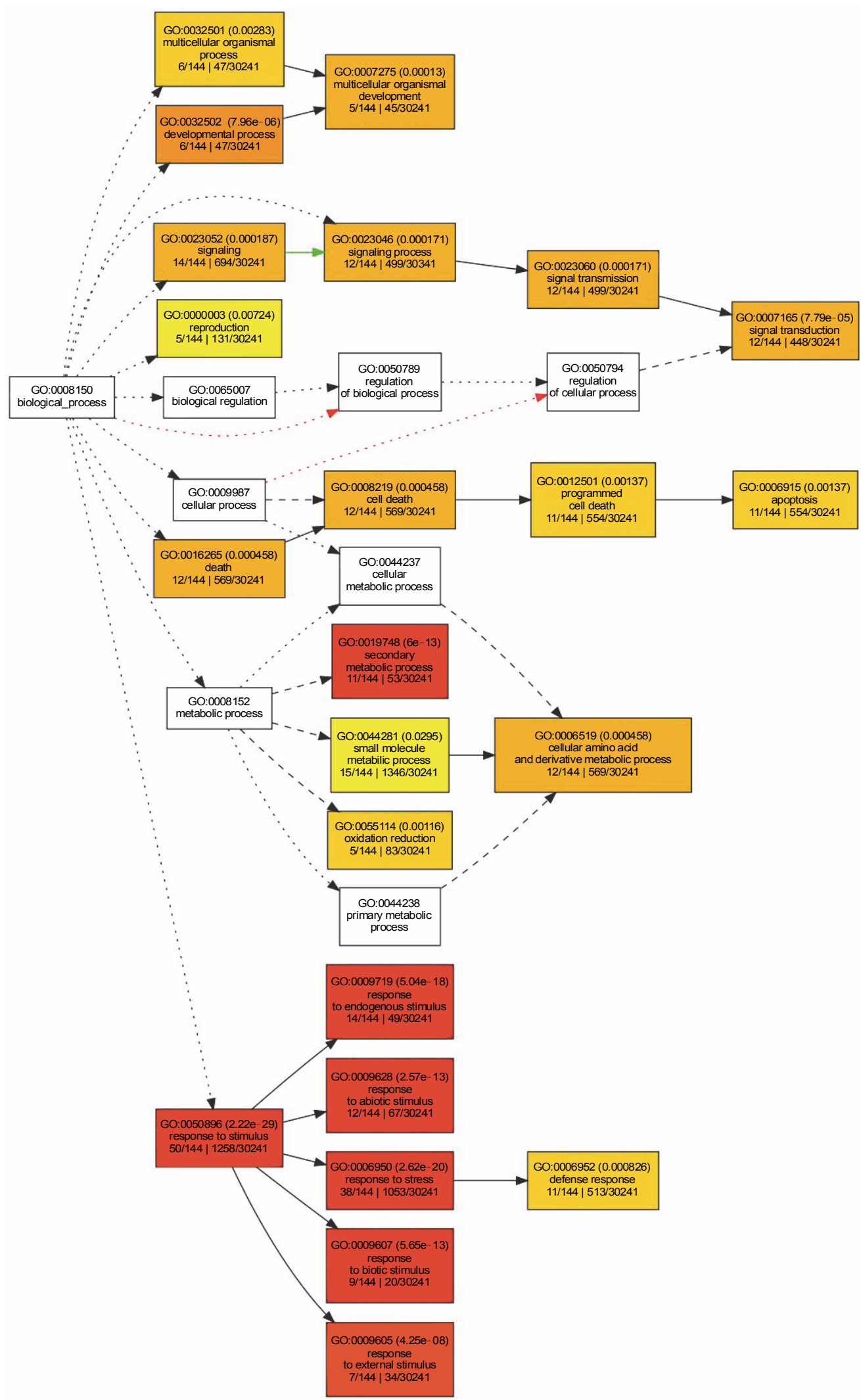

Fig. 1. Gene ontology enrichment analysis of uniquely up-regulated genes in tolerant genotype. The diagram shows GO terms of the significantly enriched biological processes determined by SEA analysis. To highlight the significance, the squares are color coded. In terms of importance degree and ranking, yellow and red are calibrated as 1 (least significance level) and 9 (most significant) respectively. GO categories are determined by squares proceeded by bracketed significance level, GO category name and gene ratio in both the list and on the microarray associated to that classification 


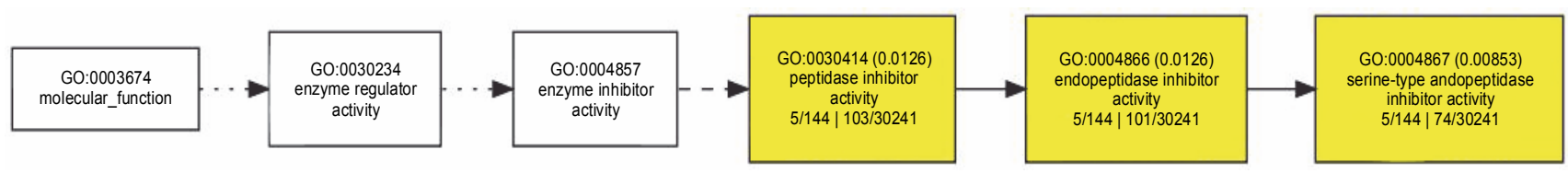

Fig. 2. ontology enrichment analysis of uniquely up-regulated genes in tolerant genotype. The diagram shows GO terms of the significantly enriched molecular functions determined by SEA analysis. To highlight the significance, the squares are color coded. In terms of importance degree and ranking, yellow and red are calibrated as 1 (least significance level) and 9 (most significant) respectively. GO categories are determined by squares proceeded by bracketed significance level, GO category name and gene ratio in both the list and on the microarray associated to that classification

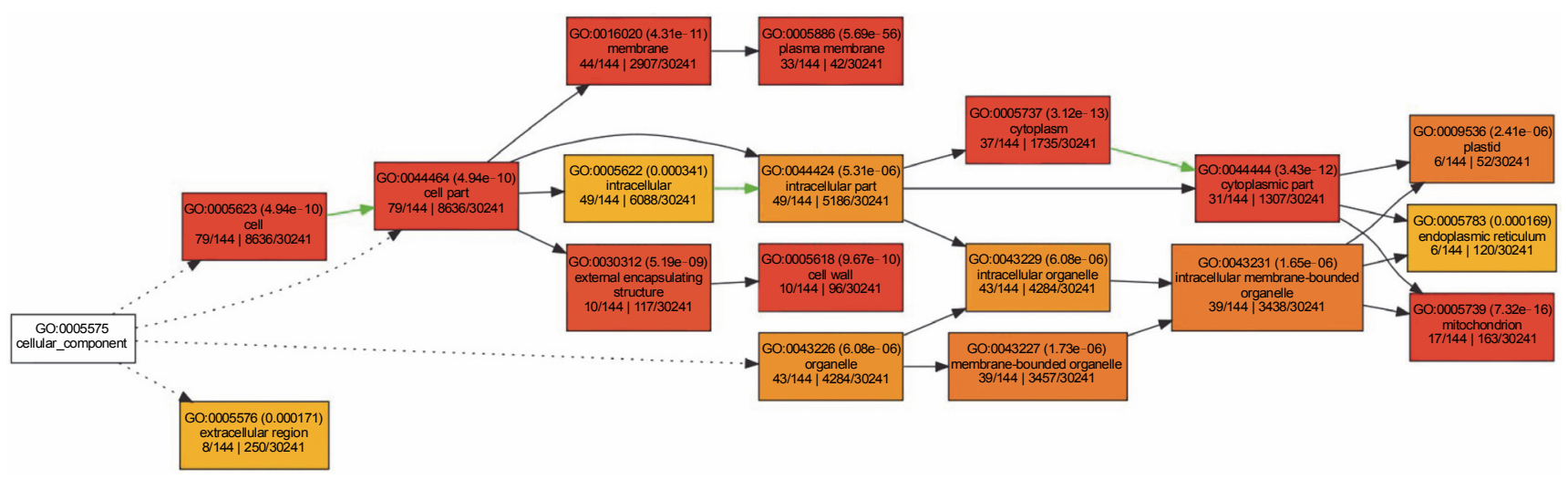

Fig. 3. Gene ontology enrichment analysis of uniquely up-regulated genes in tolerant genotype. The diagram shows GO terms of the significantly enriched cellular components determined by SEA analysis. To highlight the significance, the squares are color coded. In terms of importance degree and ranking, yellow and red are calibrated as 1 (least significance level) and 9 (most significant) respectively. GO categories are determined by squares proceeded by bracketed significance level, GO category name and gene ratio in both the list and on the microarray associated to that classification

December 12,2018sults, the involvement of Glutathione S-transferases and L-ascorbate peroxidase in biological stress response processes was explicitly indicated (Table 2). The fold-changes of the genes enriched in glutathione metabolism pathway in the drought sensitive and tolerant genotypes are presented in the Table 3 . The complete list of all the probe sets, their corresponding MSU IDs and RAP annotations, their GO terms and KEGG pathway mappings were presented in supplementary Table 1.

\section{Gene network analysis}

The network contained 120 nodes, 117 edges and 12 connected components. The excel format of the gene network, containing all relations and entities are presented as supplementary Table 2. As indicated in Figure 4, the results of gene network analysis unveiled that some genes from the following categories "involved in translational initiation” (LOC_Os01g09890, LOC_Os01g12870, LOC_Os05g39960,LOC_Os12g41400), "involved in glutathione metabolic process” (LOC_Os01g 27210), “involved intryptophanbiosynthetic process" (LOC_Os02g 16630), "involved in proteolysis" (LOC_Os03g41438, LOC_ Os09g19790), "involved in oxidation reduction" (LOC_ Os03g55240), "involved in protein glycosylation" (LOC_ Os03g60700), "involved in Golgi organization" (LOC_ Os03g63250), "involved in carbohydrate metabolic process" (LOC_Os05g37700), "involved in protein amino acid phosphorylation" (LOC_Os06g05520), and "involved in DNA repair" (LOC_Os07g30980, LOC_Os11g 40150), "involved in transmembrane transport" (LOC_ Os07g38400), and "involved in transcription factor activity" (LOC_Os08g 28214) were among uniquely upregulated genes in the tolerant genotype. Based on overall results of applying various algorithms (Table 4), LOC_Os01g09890, LOC_Os07g30980, LOC_Os08g 39140, LOC_Os03g60700, LOC_Os05g39960, LOC_ Os01g27210, and LOC_Os11g 40150 genes were considered critical in the network. The fold-changes of the critical genes identified by Cyto-Hubba in the drought sensitive and tolerant genotypes are presented in Table 3. As shown in Table 3, the fold-change of the ex- 
Table 1. KEGG pathway enrichment analysis

\begin{tabular}{|c|c|c|c|c|c|c|c|c|c|c|c|c|c|}
\hline Type & Term & Description & Count & $\begin{array}{c}\text { Percent } \\
{[\%]}\end{array}$ & $P$-value & Genes & $\begin{array}{l}\text { List } \\
\text { total }\end{array}$ & $\begin{array}{l}\text { Pop } \\
\text { hits }\end{array}$ & $\begin{array}{l}\text { Pop } \\
\text { total }\end{array}$ & $\begin{array}{c}\text { Fold } \\
\text { change }\end{array}$ & Bonferroni & Benjamini & FDR \\
\hline KEGG & osa 00480 & $\begin{array}{l}\text { glutathione } \\
\text { metabolism }\end{array}$ & 5 & 22.72 & 0.00093 & $\begin{array}{l}\text { LOC_Os01g27210 } \\
\text { LOC_Os07g28480 } \\
\text { LOC_Os07g49400 } \\
\text { LOC_Os09g29200 } \\
\text { LOC_Os10g38740 }\end{array}$ & 22 & 75 & 3614 & 10.9515 & 0.00093 & 0.00093 & 0.00093 \\
\hline
\end{tabular}

Table 2. GO annotation of genes enriched in glutathione metabolism pathway

\begin{tabular}{|c|c|c|c|c|}
\hline Gene & Description & Biological process & Molecular function & Cellular component \\
\hline LOC_Os01g27210 & similar to glutathione S-transferase GST 8 & $\begin{array}{l}\text { response to abiotic stimulus (GO:0009628); } \\
\text { response to stress (GO:0006950) }\end{array}$ & $\begin{array}{c}\text { translation factor activity; } \\
\text { RNA binding (GO:0008135); } \\
\text { transferase activity (GO:0016740) }\end{array}$ & $\begin{array}{c}\text { mitochondrion } \\
\text { (GO:0005739); } \\
\text { nucleus (GO:0005634) }\end{array}$ \\
\hline LOC_Os07g28480 & similar to glutathione S-transferase GST 19 & & transferase activity (GO:0016740) & \\
\hline LOC_Os09g29200 & similar to glutathione S-transferase GST 23 & & transferase activity (GO:0016740) & \\
\hline LOC_Os10g38740 & similar to glutathione S-transferase GST 30 & & transferase activity (GO:0016740) & \\
\hline LOC_Os07g49400 & L-ascorbate peroxidase & response to oxidative stress (GO:0006979) & $\begin{array}{c}\text { heme binding (GO:0020037); } \\
\text { catalytic activity (GO:0003824); } \\
\text { peroxidase activity (GO:0004601) }\end{array}$ & $\begin{array}{l}\text { plastid (GO:0009536); } \\
\text { cell wall (GO:0005618); } \\
\text { thylakoid (GO:0009579); } \\
\text { cytosol (GO:0005829); } \\
\text { nucleus (GO:0005634) }\end{array}$ \\
\hline
\end{tabular}


Table 3. The expression fold change of critical genes identified by Cyto-Hubba and the genes enriched in glutathione metabolism pathway in drought tolerant and sensitive genotypes under drought stress

\begin{tabular}{|c|c|c|c|c|c|c|c|c|c|c|c|}
\hline \multirow[b]{2}{*}{ Critical genes } & \multirow[b]{2}{*}{ Probeset ID } & \multicolumn{5}{|c|}{ Sensitive genotype } & \multicolumn{5}{|c|}{ Tolerant genotype } \\
\hline & & $\begin{array}{c}\text { fold } \\
\text { change }\end{array}$ & $\begin{array}{c}\text { symmetrical } \\
\text { raw } \\
\text { fold change }\end{array}$ & T statistic & $P$-value & $\begin{array}{c}\text { adjusted } \\
P \text {-value }\end{array}$ & $\begin{array}{c}\text { fold } \\
\text { change }\end{array}$ & $\begin{array}{c}\text { symmetrical } \\
\text { raw } \\
\text { fold change }\end{array}$ & T statistic & $P$-value & $\begin{array}{c}\text { adjusted } \\
P \text {-value }\end{array}$ \\
\hline LOC_Os01g09890 & Os.4960.1.S1_at & 0.3521087 & -2.840032 & 38.00805 & $3.79 \cdot 10^{-12}$ & $8.80 \cdot 10^{-10}$ & 5.816487 & 5.816487 & -10.4603 & $1.05 \cdot 10^{-6}$ & $1.39 \cdot 10^{-5}$ \\
\hline LOC_Os07g30980 & Os.9401.1.S1_at & 0.7739163 & -1.29213 & 2.883633 & 0.016284 & 0.0499 & 3.541434 & 3.541434 & -6.53353 & $6.61 \cdot 10^{-5}$ & 0.000458 \\
\hline LOC_Os08g39140 & Os.37616.1.S1_at & 0.485775 & -2.05857 & 9.302767 & $3.07 \cdot 10^{-6}$ & $4.07 \cdot 10^{-5}$ & 0.589921 & -1.69514 & 6.148791 & 0.000108 & 0.00102 \\
\hline LOC_Os03g60700 & Os.4641.1.S1_at & 0.6771128 & -1.47686 & 3.96938 & 0.002646 & 0.010456 & 3.574421 & 3.574421 & -7.59844 & $1.84 \cdot 10^{-5}$ & 0.000153 \\
\hline LOC_Os05g39960 & Os.55936.1.S1_at & 0.4764342 & -2.09893 & 8.623142 & $6.07 \cdot 10^{-6}$ & $5.26 \cdot 10^{-5}$ & 3.490252 & 3.490252 & -9.78756 & $1.93 \cdot 10^{-6}$ & $2.31 \cdot 10^{-5}$ \\
\hline LOC_Os01g27210 & Os.7911.1.S1_at & 0.6270996 & -1.59464 & 6.452954 & $7.32 \cdot 10^{-5}$ & 0.000453 & 5.142252 & 5.142252 & -12.2802 & $2.35 \cdot 10^{-7}$ & $4.07 \cdot 10^{-6}$ \\
\hline LOC_Os11g40150 & Os.57536.1.S1_at & 0.8546111 & -1.17012 & 3.668557 & 0.004327 & 0.015975 & 8.262954 & 8.262954 & -18.8518 & $3.82 \cdot 10^{-9}$ & $1.67 \cdot 10^{-7}$ \\
\hline LOC_Os07g28480 & Os.15614.1.S1_at & 0.06503069 & -15.3774 & 44.49726 & $7.90 \cdot 10^{-13}$ & $3.44 \cdot 10^{-10}$ & 13.03155 & 13.03155 & -17.811 & $6.64 \cdot 10^{-9}$ & $2.49 \cdot 10^{-7}$ \\
\hline LOC_Os09g29200 & Os.11962.1.S1_at & 0.7665262 & -1.30459 & 3.105232 & 0.011151 & 0.036172 & 3.648096 & 3.648096 & -13.8929 & $7.29 \cdot 10^{-8}$ & $1.59 \cdot 10^{-6}$ \\
\hline
\end{tabular}

Table 4. The list of critical genes identified based on overall results of applying different algorithms embedded in Cyto-Hubba including Betweenness centrality, Bottleneck (BN), Closeness centrality, Clustering coefficient, Degree, Density of Maximum Neighborhood Component (DMNC), EcCentricity, Edge Percolated Component (EPC), Maximal Clique Centrality (MCC), Maximum Neighborhood Component (MNC), Radiality centrality, Stress centrality

\begin{tabular}{|c|c|c|c|c|c|c|c|c|c|c|c|c|c|}
\hline Name & Betweenness & BottleNeck & Closeness & $\begin{array}{l}\text { Clustering } \\
\text { coefficient }\end{array}$ & Degree & DMNC & EcCentricity & $\mathrm{EPC}$ & $\mathrm{MCC}$ & $\mathrm{MNC}$ & Radiality & Stress & $\begin{array}{l}\text { The number } \\
\text { of algorithms } \\
\text { that identified } \\
\text { critical genes }\end{array}$ \\
\hline LOC_Os01g09890 & 1300 & 44 & 28.66667 & 0.083 & 23 & 0.10967 & 0.12222 & 14.607 & 0 & 22 & 1.15116 & 1300 & 8 \\
\hline LOC_Os07g30980 & 1375 & 41 & 30.75 & 0.07407 & 27 & 0.10222 & 0.08542 & 17.541 & 0 & 26 & 1.88771 & 1970 & 8 \\
\hline LOC_Os08g39140 & 1148 & 44 & 23 & 0 & 3 & 0 & 0.18333 & 12.566 & 2 & 1 & 1.16822 & 1148 & 7 \\
\hline LOC_Os03g60700 & 936 & 44 & 24 & 0.11667 & 16 & 0.14021 & 0.12222 & 11.772 & 1 & 15 & 1.03178 & 936 & 7 \\
\hline LOC_Os05g39960 & 546 & 8 & 20 & 0.17778 & 10 & 0.19093 & 0.12222 & 9.675 & 1 & 9 & 0.92946 & 546 & 4 \\
\hline LOC_Os01g27210 & 90 & 11 & 10 & 0.15152 & 12 & 0.16968 & 0.09167 & 5.971 & 0 & 11 & 0.21083 & 90 & 4 \\
\hline LOC_Os11g40150 & 300 & 5 & 20.28333 & 0.25 & 8 & 0.25611 & 0.06833 & 13.14 & 0 & 7 & 1.63146 & 388 & 4 \\
\hline
\end{tabular}



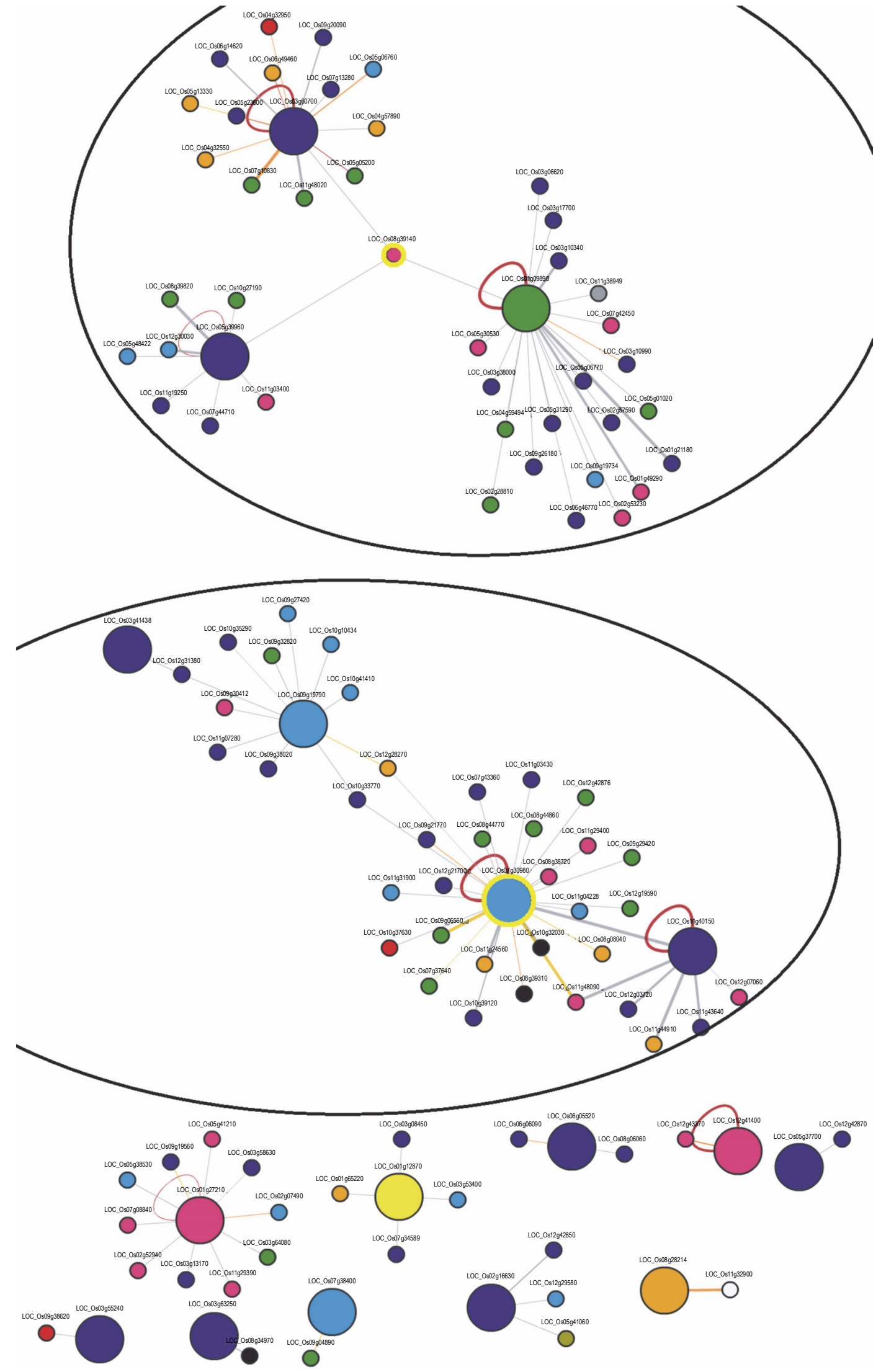

Fig. 4. The interaction network of uniquely upregulated genes in tolerant genotype. The web based tool 'Rice Interactions Viewer' (http://bar.utoronto.ca/interactions/cgi-bin/rice_interactions_viewer.cgi) was used to generate the interaction network. Two important candidates: LOC_Os07g30980 (uvrD/REP helicase family protein) and LOC_OS08g39140 (heat shock protein) were highlighted. Two major subnetworks within the network were indicated by circles. Large circles include genes that were among those used as queries in creating network. For each edge which connects a pair of proteins, different color is based on the coexpression value. As well the relative thickness corresponds to its Confidence Value 
pression of gene LOC_Os08g39140 was below the cutoff used for the tolerant genotype; LOC_ OS08g39140 was not among 308 probe sets uniquely upregulated in the tolerant rice genotype. However, according to network analysis, LOC_OS08g39140 joins the 3 major nodes, which are uniquely upregulated in a tolerant genotype, together. Also, LOC_OS08g39140 was identified as a critical gene by 7 out of 12 algorithms of cytoHubba. Based on our microarray data analysis, LOC_OS08g 39140 expression level was decreased in both tolerant and sensitive genotypes under drought stress (Table 3). However, the amount of decrease in the expression level of LOC_OS08g39140 in the tolerant genotype was smaller than in sensitive genotype under drought stress, supporting its critical role in conferring the drought tolerance.

Among the genes in the network, LOC_Os07g30980 (uvrD/REP helicase family protein, putative, expressed), located at the center of network, has the highest number of interactions with other genes. On the other hand, acting like an intermediary agent, LOC_OS08g39140 (heat shock protein, putative, expressed) joins the 3 major nodes including LOC_Os01g09890 (translation initiation factor SUI1, putative, expressed), LOC_Os05g39960 (40S ribosomal protein S26, putative, expressed), and LOC_Os03g60700 (dolichyl-phosphate beta-glycosyltransferase, putative, expressed) together. Biological process enrichment analysis of the genes forming the network suggested that 18 genes enriched in response to stress stimulus. Five genes (out of these 18 genes) including LOC_Os11g40150 (DNA repair protein Rad51), LOC_Os07g30980 (uvrD/REP helicase family protein), LOC_Os03g55240 (cytochrome P450), LOC_Os06g 05520 (OsMKK1), and LOC_Os01g27210 (glutathione S-transferase) were among uniquely upregulated genes in the tolerant genotype. Notably, 10 out of these 18 genes were enriched in GO term "response to abiotic stress stimulus”, including LOC_Os03g55240 (cytochrome P450, putative, expressed), LOC_Os08g44770 (copper/zinc superoxide dismutase, putative, expressed), LOC_Os01g 27210 (glutathione S-transferase, putative, expressed), LOC_Os09g30412 (heat shock protein, putative, expressed), LOC_Os08g39140 (heat shock protein, putative, expressed), LOC_Os05g38530 (DnaK family protein, putative, expressed), LOC_Os11g40150 (DNA repair protein Rad51, putative, expressed), LOC_Os02g07490 (glyceraldehyde-3-phosphate dehydrogenase, putative, expres- sed), LOC_Os06g05520 (OsMKK1 - putative MAPKK based on amino acid sequence homology, expressed), LOC_Os05g41210 (OsCam2 - Calmodulin, expressed).

\section{Promoter analysis}

Analysis of promoter regulatory elements can provide valuable opportunity for prediction of gene functions. Various regulatory elements connected with the plant responses to abiotic and biotic stresses including ABRE (cis-acting element involved in the abscisic acid responsiveness), CCAAT-box (MYBHv1 binding site), CGTCAmotif (cis-acting regulatory element involved in the MeJA-responsiveness), MBS (MYB binding site involved in drought-inducibility), TC-rich repeats (cis-acting element involved in defense and stress responsiveness), TGACG-motif (cis-acting regulatory element involved in the MeJA-responsiveness), motif IIb (abscisic acid responsive element), HSE (cis-acting element involved in heat stress responsiveness), P-box (gibberellin-responsive element), TCA-element (cis-acting element involved in salicylic acid responsiveness) were predicted in 2 analyzed promoter sequences of LOC_Os07g30980 and LOC_OS08g39140. The numbers of identified Cis-Acting Regulatory Elements are given in Table 5.

\section{Expression analysis of critical genes in rice various organs and developmental stages}

It has been found that LOC_Os07g30980 (uvrD/REP helicase family protein, putative, expressed) and LOC_ OS08g39140 (heat shock protein, putative, expressed) were expressed in all rice tissues and at its all developmental stages. The highest LOC_Os07g30980 expression level was found in stamen, spikelet, and panicle. According to the expression analysis of LOC_Os07g 30980 at various rice developmental stages, the highest expression level of LOC_Os07g30980 was noticed in booting stage, and flowering stage followed by heading stage. Also, the highest expression level of LOC_OS08g 39140 was found in root tip, pistil, and root. According to expression analysis of LOC_OS08g39140 in various rice developmental stages, the highest expression level of LOC_OS08g39140 was noticed in stem elongation stage.

\section{Discussion}

The data series (GSE21651) of public rice microarray data (from NCBI gene expression omnibus) provided by Rai and Singh (2011) was analyzed. The gene series 
Table 5. Numbers of identified Cis-Acting Regulatory Elements in the promoter regions of LOC_Os07g30980 and LOC_Os08g39140

\begin{tabular}{|c|c|c|c|}
\hline & LOC_Os07g30980 & LOC_Os08g39140 & \\
\hline $\mathrm{ABRE}$ & 3 & 0 & $\begin{array}{l}\text { cis-acting element involved } \\
\text { in the abscisic acid responsiveness }\end{array}$ \\
\hline CCAAT-box & 1 & 0 & MYBHv1 binding site \\
\hline CGTCA-motif & 1 & 1 & $\begin{array}{l}\text { cis-acting regulatory element involved } \\
\text { in the MeJA-responsiveness }\end{array}$ \\
\hline MBS & 3 & 1 & MYB binding site involved in drought-inducibility \\
\hline TC-rich repeats & 2 & 1 & $\begin{array}{l}\text { cis-acting element involved } \\
\text { in defense and stress responsiveness }\end{array}$ \\
\hline TGACG-motif & 1 & 1 & $\begin{array}{l}\text { cis-acting regulatory element involved } \\
\text { in the MeJA-responsiveness }\end{array}$ \\
\hline motif IIb & 3 & 0 & abscisic acid responsive element \\
\hline HSE & 0 & 2 & $\begin{array}{l}\text { cis-acting element involved } \\
\text { in heat stress responsiveness }\end{array}$ \\
\hline P-box & 0 & 1 & gibberellin-responsive element \\
\hline TCA-element & 0 & 1 & $\begin{array}{l}\text { cis-acting element involved } \\
\text { in salicylic acid responsiveness }\end{array}$ \\
\hline
\end{tabular}

(GSE21651) included GSM540080, GSM540081, GSM540082, GSM540083, GSM540084, GSM540085, GSM540086, and GSM540087. In the given experiment, 8 GeneChip arrays were used for 8 RNA samples extracted from 2 biological replicates of 2 rice genotypes IR64 (drought-sensitive) and Vandana (drought-tolerant), each grown under control and drought stress conditions (Rai and Singh, 2011). Eight downloaded CEL files of 3' expression arrays were imported into Affymetrix expression console software and normalized by robust multichip analysis (RMA) algorithm. The analyses have been carried out by FlexArray package (McGill University, Canada). Meanwhile to select the genes with significant differential expression at corrected $P$-value $\leq 0.05$, the Bayesian twosample $T$-test in FlexArray was used through which a fold change of gene expression was determined. Accordingly, the genes with a significant differential expression (corrected $P$-value $\leq 0.05$ ) and remarkably upregulated (equal to or greater than 3 -fold change) in a tolerant genotype and downregulated (less than 1-fold change) in a sensitive one under drought stress were selected (Zinati, 2017).

\section{GO enrichment analysis}

GO enrichment analysis was performed on 308 probe sets uniquely upregulated in tolerant rice genotype to characterize the critical functions and pathways these probe sets are involved in. GO enrichment analysis was also utilized to find enriched GO terms in biological process, molecular function, and cellular component in drought stress responses in rice. In terms of "biological process", significantly upregulated biological processes in the tolerant rice genotype were related to GO terms such as developmental process ( 5 genes), signaling (14 genes), reproduction (5 genes), cell death (12 genes), cellular amino acid and derivative metabolic process (12 genes), oxidation reduction (5 genes), secondary metabolic process (11 genes), response to stimulus (50 genes). According to the results, GO term enrichment analysis revealed that most of the uniquely upregulated genes (50 genes) participated in responses to stimulus. Biological process enrichment analysis revealed that the response to abiotic stress stimulus is highly overrepresented in the tolerant genotype in drought stress conditions. Supporting these GO enrichment results, cellular amino acid and derivative metabolic process, secondary metabolic process, signaling, cell death, and response to stimulus biological categories have been previously reported in a study conducted by Moumeni and coworkers (2013) on 2 pairs of drought-tolerant and susceptible rice nearisogenic lines under drought stress.

These GO categories notably include genes that were earlier reported to be associated with drought tolerance 
(Kumar et al., 2008; Khong et al., 2015; Batth et al., 2017). For instance, in the "developmental process" biological category, LOC_Os08g02070 (OSMADS26) was reported to have a possible function related to water deficit in rice (Khong et al., 2015). LOC_Os06g37150 (L-ascorbate oxidase; OsAAO2) which fell into "oxidation reduction" biological category was revealed to be the most stress responsive gene in response to salinity and drought stresses (Batth et al., 2017).

It is well distinguished that drought stress perception, signal transduction leading to molecular, cellular and whole plant adaptive processes are critical steps toward achieving drought tolerance (Lenka et al., 2011). In "signaling" biological category, several genes such as LOC_Os06g06760 (protein kinase, putative, expressed), LOC_Os01g49614 (Protein kinase domain containing protein, expressed), LOC_Os04g01320 (serine/threonine-protein kinase receptor precursor, putative, expressed), LOC_Os04g30330 (OsWAK59 - OsWAK receptorlike cytoplasmic kinase OsWAK-RLCK), LOC_Os04g 30260 (OsWAK47 - OsWAK receptor-like protein kinase), LOC_Os01g10430 (protein kinase family protein, putative, expressed), LOC_Os11g46860 (wall-associated receptor kinase-like 4 precursor, putative, expressed), and LOC_Os06g05520 (OsMKK1 - putative MAPKK based on amino acid sequence homology, expressed) were found to encode families of protein kinases. Engaged in the processes of biotic and abiotic stress detection and their relative signal transduction, mitogen-activated protein kinase (MAPK) pathways are among one of the most studied intracellular signaling ones (Mishra et al., 2006; Jaggi, 2018; Wimalasekera and Scherer, 2018).

MAPK cascades consist of 3 components, a MAP kinase kinase kinase (MEKK/MAPKKK), a MAP kinase kinase (MKK/MAPKK), and a MAP kinase (MPK/MAPK), that are sequentially activated by a phosphorylation cascade (Mishra et al., 2006). In the rice (Oryza sativa L.) genome, 17, 8, and 75 genes have been annotated as MAPKs, MAPKKs, MAPKKKs, respectively (Rao et al., 2010; Hamel et al., 2006).

Despite Sreenivasulu and coworkers (2007), maintained that the transcriptome engineering (utilizing master switch genes like transcription factors and signaling proteins) has the potential to boost the crop plants' abiotic stress tolerance, most components of the key sensing and signal transduction mechanisms for drought tolerance have not been identified. In "signal transduc- tion" biological category, 12 genes (LOC_Os08g 10250, LOC_Os06g06760, LOC_Os01g49614, LOC_Os05g 25350, LOC_Os06g07923, LOC_Os05g11810, LOC Os04g30330, LOC_Os04g30260, LOC_Os11g 47920, LOC_Os01g10430, LOC_Os11g46860, LOC_Os06g 05520) were pinpointed. LOC_Os06g05520 (OsMKK1), among the genes enriched signal transduction, was notably one of the genes forming the network. Besides, the presence of other diverse GO terms for LOC_Os06g 05520 including response to stress (G0:0006950), response to abiotic stress (GO:0009628), protein amino acid phosphorylation (0006468), cellular macromolecule metabolic process (GO:0044260), response to external stimulus (GO:0009605) corroborated the significance of the gene in drought stress response.

Importantly, the OsMKK1 transcript level was markedly increased due to drought stress conditions and might thereby be involved in drought stress signaling (Kumar et al., 2008). Wang and coworkers (2014) unveiled the involvement of OsMKKI in a signaling pathway which manages salt stress tolerance in rice. As an integral component of MAPK cascade, MKKs take on a crucial role in incorporating the upstream signals from MAPKKKs and transferring signals to downstream MPKs for more expected responses from cells (Kumar et al., 2008). Moreover, regarding salt response, it was demonstrated that OsMPK4 is one of OsMKK1's downstream targets (Wang et al., 2014).

Within the "molecular function" category, upregulated genes were enriched in "serine-type endopeptidase inhibitor activity". Regarding the upregulation of those genes in the tolerant rice genotype subjected to drought stress, one can conclude that the tolerant genotype would have significantly higher serine-type endopeptidase inhibitor activity than sensitive genotype. Consistently with the molecular function enrichment results, serine-type endopeptidase inhibitor activity (GO:0004867) has been previously reported in a study conducted by Batista and coworkers (2017) on effects of salinity on the transcriptomes of 3 rice lines (Moumeni et al., 2013). Peptidase inhibitor activity in plants is mostly related to the response to environmental stresses, plant protection against insects, and pathogens (Habib et al., 2007)

Likewise, cellular component enrichment analysis showed that most uniquely upregulated genes were localized in extracellular regions, intracellular membranebounded organelles such as plastids, endoplasmic reti- 
culum, as well as mitochondria, plasma membranes and cell walls.

Furthermore, given the large variety in drought stress responses in plants, it was noticed through cellular component enrichment analysis, that the importance of intracellular membrane-bound organelles, plasma membranes, and cell walls was highlighted. The primary sites for the production of ROS as a signaling molecule are some intracellular organelles such as chloroplasts and mitochondria which, at the same time, represent a remarkable capacity in their production (Dat et al., 2000; Van Breusegem et al., 2001). It is worth noting that most of the receptor proteins are located in plasma membrane, which is therefore directly involved in the stress sensing.

\section{KEGG pathway enrichment analysis}

To comprehend the mechanisms of stress tolerance, recognizing significant pathways which are changed in response to stress conditions demands priority (Lenka et al., 2011). In a detailed study of 1-week old rice (Oryza sativa L. var. IR64) seedlings, pathways such as cytokinin and secondary metabolites, cell wall loosening and structural components, cholesterol and very long chain fatty acid, and osmoprotectants (amino acid and quaternary ammonium compounds, polyols and sugars) metabolisms were notably influenced by water-deficit stress conditions (Ray et al., 2011). Interestingly, the engineering of metabolic pathways is now being increasingly used to improve stress tolerance in plants (Prakash et al., 2014).

In the present study, KEGG pathway enrichment analysis unraveled uniquely upregulated genes significantly enriched in glutathione metabolism pathway. Five genes including Glutathione S-transferases and L-ascorbate peroxidase were enriched in glutathione metabolism pathway. Based on the overview of functionally characterized genes in rice online database (OGRO) (Yamamoto et al., 2012) of 5 genes identified in glutathione metabolism pathway, the function of LOC_Os07g 49400 has been characterized and reported to be related to oxidative stress resistance (Bonifacio et al., 2011). Furthermore, among genes enriched in glutathione metabolism pathway, LOC_Os01g27210 (Like Glutathione S-transferase GST 8) was also one of the genes forming the network. Regarding the actual role of Glutathione S-transferases (GSTs) in responding to drought conditions, the relative reports are limited (Labudda and Safiul Azam, 2014). Subjected to water shortage, drought tolerant wheat genotypes preceded sensitive ones in terms of detecting the induction of GST activity (Gallé el al. 2009). In addition, a number of studies have reported that GSTs over-expression resulted in improving the plants drought tolerance. For instance, transgenic tobacco overexpressing a chloroplastic GST from Prosopis juliflora (George et al., 2010) and a tau class of the GST gene, GsGST from wild soybean (Glycine soja) (Ji et al., 2010) displayed enhanced dehydration tolerance. In another study, over-expression of tau glutathione transferases stemming from Citrus sinensis (CsGSTU1 and CsGSTU2) in transgenic tobacco demonstrated both salinity and drought tolerance (Cicero et al., 2015). According to Roxas and coworkers (2000), overexpression of a tobacco glutathione S-transferase with glutathione peroxidase activity in transgenic tobacco (Nicotiana tabacum L.) enhanced ROS-scavenging capacity. This protective effect appears to attenuate cellular damage and allows seedlings to maintain high growth and metabolic activity levels under a variety of stressful conditions (Roxas et al., 2000).

The cytosolic ascorbate peroxidase 2 (OSAPX2) was reported to play a critical role in growth and reproduction in rice subjected to drought, salt and cold stresses by protecting the rice seedlings against abiotic stresses via reactive oxygen species scavenging (Zhang et al., 2013). Overexpression of $O S A P X 2$ increased the salt tolerance of transgenic Arabidopsis and Medicago sativa plants (Lu et al., 2007; Guan et al., 2012).

In the current study, the upregulation of glutathione metabolism pathway components including glutathione S-transferases and L-ascorbate peroxidase supported the involvement of GSTs and L-ascorbate peroxidase in response to drought stress. Bearing in mind that a variety of abiotic stresses drive the plants to display ROS (reactive oxygen species) overproduction which in turn results in incurring damage to almost entire cellular macromolcules such as DNA, lipids, proteins and carbohydrates, the ultimate oxidative stress emergence is not unpredictable. However, plants are equipped with enzymatic (e.g. APX, ascorbate peroxidase; GlutathioneS-transferanse, GST) and non-enzymatic antioxidant defense systems that consolidate in exercising control over the cascades of turbulent and unmanageable oxidation and thus shield plants against such damages through 
searching for ROS (Gill and Tuteja, 2010). It should be taken into consideration that most effective metabolic pathways are the ones engaged in detoxification of ROS generated during the abiotic stress conditions (Prakash et al., 2014).

According to our results, genes encoding important enzymes involved in the glutathione antioxidant pathway were upregulated in the tolerant genotype under drought stress, indicating the importance of antioxidant defense in drought stress tolerance. From the genes enriched in glutathione metabolism pathway, LOC_ Os01g27210 (glutathione S-transferase) and LOC_Os07g 49400 (OsAPX2 - Cytosolic Ascorbate Peroxidase encoding gene 4,5,6,8) also enriched the response to stress stimulus of biological process category substantiating the notion that the more the genes in charge of the production of enzymes involved in ROS scavenging are modified, the higher chance to improve the abiotic stress tolerance (Prakash et al., 2014).

\section{Gene network analysis}

To recognize the available networks in tolerant genotype that are specifically active when exposed to drought stress, network analysis is conducted. Network topology, on the other hand, can underline genes which show remarkably higher connection (central ones) in addition to those connecting various sub-networks. Moreover, the critical genes detected by Cyto-Hubba may serve as candidate genes for increasing drought tolerance. The subnetworks of these critical nodes might provide the opportunity of a more precise understanding of the biological processes underlying drought tolerance. Two major subnetworks found within the network are indicated by circles in Figure 4. The first subnetwork is enriched in ribosome (osa03010) (10 genes), N-glycan biosynthesis (osa00510) (5 genes), and protein processing in endoplasmic reticulum (osa04141) (6 genes) pathways. Some genes in this subnetwork are involved in biological processes such as translation, and protein glycosylation. The second subnetwork is enriched in pyrimidine metabolism (osa00240) (3 genes), homologous recombination (osa03440) (2 genes), and glutathione metabolism (osa00480) (2 genes) pathways. Some genes in this subnetwork are involved in biological processes such as response to stress, DNA metabolic process, carbohydrate metabolic process, and DNA repair. LOC_Os07g30980 (uvrD/REP helicase family pro- tein, putative, expressed) present at the second subnetwork was identified as a critical gene by 8 out of 12 algorithms. LOC_Os07g30980 is located at the center of network and has the highest number of interactions with other genes. Based on the analysis of the LOC_Os07g 30980 in STRING, the network was enriched in genes associated with the homologous recombination (osa03440), mismatch repair (osa03430) and ubiquitin mediated proteolysis (osa04120). The optimal growth in plant, productivity and development are directly linked to the stability of the genome (Tuteja and Tuteja, 2013). Drought as an abiotic stress which induces some metabolic byproducts like ROS, can make the plants prone to DNA damage which, accordingly, results in instability of the genome (Gill and Tuteja, 2010). Such DNA damage, in its own turn, is the source of sequential turbulence in the functionality of the plant leading to the destruction of cell membranes, protein synthesis reduction and photosynthetic protein damage, all of which can cause detrimental effects on both development and growth of the whole organism (Britt, 1999). The DNA impairment can precipitate induction or arrest transcription, induction of signal transduction pathways, cell membrane destruction, replication errors and genomic instability (Britt, 1999; Cooke et al., 2003). To restore stability to the genome, the mismatch repair (MMR) system is employed which is a prime pathway for DNA repair and an integral component, of which UvrD helicase takes on a crucial role in supporting the unwinding task (Tuteja and Tuteja, 2013). According to network analysis results, uvrD/REP helicase family protein can be assumed to play critical function in drought tolerance. To the best of our knowledge, the role of LOC_Os07g30980 in drought tolerance in rice has not been reported previously. This, thereupon, may ignite interest in opening new venues of knowledge for the role of the enzyme in drought tolerance. Additionally, LOC_OS08g39140 (heat shock protein, putative, expressed) was another gene that was identified as critical gene by 7 out of 12 algorithms. LOC_OS08g39140 joins the 3 major nodes including LOC_Os01g09890 (translation initiation factor SUI1, putative, expressed), LOC_Os05g39960 (40S ribosomal protein S26, putative, expressed), and LOC_Os03g 60700 (dolichyl-phosphate beta-glycosyltransferase, putative, expressed) together supporting its potential efficacy in the drought tolerance. Based on the analysis of the LOC_OS08g39140 in STRING, the network was 
enriched in genes associated with the protein processing in endoplasmic reticulum (osa04141), endocytosis (osa04144) and spliceosome (osa03040). Protein-processing in the ER and degradation-related proteasome were the prevailing upregulated pathways in the endosperm of rice in ER stress response. Most of the upregulated genes involved in protein-processing in the ER encode chaperons that expedite folding, sorting, or degradation, alleviating ER stress (Qian et al., 2015). Recent evidence suggests linking of ER stress signaling to drought tolerance (Xiang et al., 2017). Environmental stress conditions, such as salt and heat stress, lead to accumulation of unfolded or misfolded proteins in endoplasmic reticulum (ER), and this subsequently provokes an unfolded protein response (UPR) or ER stress signaling to generate protein-folding factors or other factors to facilitate the folding or cleaning of proteins (Howell, 2013). GO annotation indicated that LOC_OS08g39140 resides in reticulum membrane and is involved in biological processes such as response to abiotic stress and protein folding. Moreover, LOC_OS08g39140 as well as the gene in its vicinity, LOC_OS08g39100 (encoding protein phosphatase $2 \mathrm{C}$ ) were previously reported to fall within the important drought stress related QTL (quantitative trait locus) (qsla8.1) in the marker intervals RM404RM447. The QTL detected in this marker intervals accommodate the genes handling majority of water use efficiency related traits specific leaf area and leaf width (Roja et al., 2016, 2014).

To close the loop, according to promoter analysis, the presence of $4 \mathrm{MYB}, 3 \mathrm{ABRE}$, and 3 IIb motifs in the promoter region of LOC_Os07g30980 and 1 MYB motif in the promoter region of LOC_OS08g39140 may support the notion that these critical genes might play roles in drought tolerance. MBS has been reported to be involved in the response to drought and required for the binding of MYB transcription factors (Gao et al., 2010). Also, ABRE has been shown to be necessary for perceiving ABAmediated osmotic stress signals (Fujita et al., 2011).

\section{Conclusion}

GO enrichment and pathway analyses are powerful tools for investigating the association of differentially expressed genes with biological processes and molecular functions. GO enrichment, pathway enrichment and transcriptomic-based network analyses of rice genotypes with contrasting response to drought stress can shed light on those differential mechanisms, which tolerant genotypes employ to cope with drought stress. This study analyzed uniquely upregulated genes in the tolerant rice genotype using GO enrichment, pathway enrichment and gene network analyses to unravel key biological processes, molecular functions, pathways and central genes which can attend to cope with drought stress. GO enrichment analysis suggested that drought tolerance of tolerant genotype was probably related to enhanced developmental process, signaling, reproduction, cell death, cellular amino acid and derivative metabolic process, oxidation reduction, secondary metabolic process, and response to stimulus, whereas drought sensitivity of sensitive genotype was governed by significant down-regulation of the genes involved in these biological processes. It is noteworthy that among identified GO terms, the genes involved in "response to stimulus" were most abundant, numbering 50. KEGG pathway enrichment analysis revealed the glutathione metabolism as the key pathway in the tolerant genotype imposed to drought stress. Thereby, glutathione metabolism pathway modification is supposed to be a significant research objective for the involvement of some of its components in ROS detoxification which was engendered when exposed to abiotic stress conditions. According to network analysis, tolerance of the tolerant genotype seems to be related to high DNA repair system efficiency. Taken together, these results indicated genes enriched in key GO terms, glutathione antioxidant pathway, as well as central genes in network such as LOC_Os07g30980 (uvrD/REP helicase family protein, putative, expressed) and LOC_OS08g39140 (heat shock protein, putative, expressed) would be useful candidates to obtain rice varieties with increased drought tolerance. This study provides a more reliable approach for selecting appropriate genes and pathways which may significantly contribute to develop drought-tolerant rice varieties.

\section{Supplementary material}

Supplementary 1 (http://s9.picofile.com/file/8318684684/ supplementry_1.xlsx.html)

Supplementary 2 (http://s8.picofile.com/file/8318685100/ supplementary_2.xlsx.html)

\section{Acknowledgments}

We would like to greatly thank Department of Agroecology of Agriculture and Natural Resources of Darab for supporting this research. 


\section{References}

Ahn H., Jung I., Shin S.J. et al. (2017) Transcriptional network analysis reveals drought resistance mechanisms of AP2/ERF transgenic rice. Front Plant Sci. 8: 1044.

Batth R., Singh K., Kumari S., Mustafiz A. (2017) Transcript profiling reveals the presence of abiotic stress and developmental stage specific ascorbate oxidase genes in plants. Front Plant Sci. 8: 198.

Bohnert H.J., Ayoubi P., Borchert C., Bressan R.A., Burnap R. L., Cushman J.C. et al. (2001) A genomics approach towards salt stress tolerance. Plant Physiol. Biochem. 39: 295-311.

Bonifacio A., Martins M.O., Ribeiro C.W., Fontenele A.V., Carvalho F.E., Margis-Pinheiro M., Silveira J.A. (2011) Role of peroxidases in the compensation of cytosolic ascorbate peroxidase knockdown in rice plants under abiotic stress. Plant Cell Environ. 34: 1705-1722.

Borah P., Sharma E., Kaur A., Chandel G., Mohapatra T., Kapoor S., Khurana J.P. (2017) Analysis of drought-responsive signalling network in two contrasting rice cultivars using transcriptome-based approach. Sci Rep. 7: 42131.

Britt A.B. (1999) Molecular genetics of DNA repair in higher plants. Trends Plant Sci. 4: 20-25.

Chin C.H., Chen S.H., Wu H.H., Ho C.W., Ko M.T., Lin C.Y. (2014) CytoHubba: identifying hub objects and subnetworks from complex interactome. BMC Syst. Biol. 8 (Suppl 4): S11.

Cicero L.L., Madesis P., Tsaftaris A., Piero A.R.L. (2015) Tobacco plants over-expressing the sweet orange tau glutathione transferases (CSGSTUs) acquire tolerance to the diphenyl ether herbicide fluorodifen and to salt and drought stresses. Phytochem. 116: 69-77.

Cooke M.S., Evans M.D., Dizdaroglu M., Lunec J. (2003) Oxidative DNA damage: mechanisms, mutation, and disease. FASEB J. 17: 1195-1214.

Cruts M., Theuns J., Van Broeckhoven C. (2012) Locus-specific mutation databases for neurodegenerative brain diseases. Hum Mutat. 33: 1340-1344.

Dat J., Vandenabeele S., Vranová E., VanMontagu M., Inzé D., Van Breusegem F. (2000) Dual action of the active oxygen species during plant stress responses. Cell Mol. Life Sci. 57: 779-795.

Du Z., Zhou X., Ling Y., Zhang Z., Su Z. (2010) AgriGO: a GO analysis toolkit for the agricultural community. Nucl. Acids Res. 38: W64-W70.

Farooq M., Wahid A., Lee D., Ito O., Siddique K. (2009) $A d$ vances in drought resistance of rice. CRC Crit. Rev. Plant Sci. 28(4): 199-217.

Fujita Y., Fujita M., Shinozaki K.,Yamaguchi-Shinozaki K. (2011) ABA-mediated transcriptional regulation in response to osmotic stress in plants. J. Plant Res. 124: 509-525.

Gallé A., Csiszár J., Secenji M., Guóth A., Cseuz L., Tari I., et al. (2009) Glutathione transferase activity and expression patterns during grain filling in flag leaves of wheat genotypes differing in drought tolerance: response to water deficit. J. Plant Physiol. 166(17): 1878-1891.
Gao F., Xiong A., Peng R., Jin X., Xu J., Zhu B., Chen J., Yao Q. (2010) OsNAC52, a rice NAC transcription factor, potentially responds to $A B A$ and confers drought tolerance in transgenic plants. PCTOC 100: 255.

George S., Venkataraman G., Parida A.A. (2010) Chloroplastlocalized and auxin-induced glutathione S-transferase from phreatophyte Prosopis juliflora confer drought tolerance on tobacco. J. Plant Physiol. 167(4): 311-318.

Gill S.S., Tuteja N. (2010) Reactive oxygen species and antioxidant machinery in abiotic stress tolerance in crop plants. Plant Physiol. Biochem. 48: 909-930.

Goodstein D.M., Shu S, Howson R., Neupane R., Hayes R.D., Fazo J., Mitros T., Dirks W., Hellsten U., Putnam N., Rokhsar D.S. (2012) Phytozome: a comparative platform for green plant genomics. Nucl. Acids Res. 40 (D1): D1178-D1186.

Guan Q., Takano T., Liu S. (2012) Genetic transformation and analysis of rice OsAPX2 gene in Medicago sativa. PLoS One 7: e41233.

Habib H., Fazili K.M. (2007) Plant protease inhibitors: a defense strategy in plants. BMBR 2: 68-85.

Halpin C. (2005) Gene stacking in transgenic plants - the challenge for 21st century plant biotechnology. Plant Biotechnol. J. 3: 141-155.

Hamel L.P., Nicole M.C., Sritubtim S., Morency M.J., Ellis M., Ehlting J., Ellis B.E. (2006) Ancient signals: comparative genomics of plant MAPK and MAPKK gene families. Trends Plant Sci. 11: 192-198.

Ho C.L., Wu Y., Shen H.B., Provart N.J., Geisler M. (2012) A predicted protein interactome for rice. Rice (NY) 5(1): 15.

Howell S.H. (2013) Endoplasmic reticulum stress responses in plants. Annu. Rev. Plant Biol. 64: 477-499.

Jaggi M. (2018) Recent advancement on map kinase cascade in biotic stress. [in:] Molecular Aspects of Plant-Pathogen Interaction. Ed. Singh A., Singh I., Springer Singapore: 139-158.

Ji W., Zhu Y., Li Y., Yang L., Zhao X., Cai H. et al. (2010) Overexpression of a glutathione S-transferase gene, GsGST, from wild soybean (Glycine soja) enhances drought and salt tolerance in transgenic tobacco. Biotechnol. Lett. 32(8): 1173-1179.

Khong G.N., Pati P.K., Richaud F., Parizot B., Bidzinski P., Mai C.D., Bčs M., Bourrié I., Meynard D., Beeckman T., Selvaraj M.G., Manabu I., Genga A.M., Brugidou C., Nang Do V., Guiderdoni E., Morel J.B., Gantet P. (2015) OSMADS26 negatively regulates resistance to pathogens and drought tolerance in rice. Plant Physiol. 169(4): 2935-2949.

Kumar K., Rao K.P., Sharma P., Sinha A.K. (2008) Differential regulation of rice mitogen activated protein kinase kinase (MKK) by abiotic stress. Plant Physiol. Biochem. 46: 891-897.

Labudda M., Safiul Azam F.M. (2014) Glutathione-dependent responses of plants to drought: a review. Acta Soc. Bot. Pol. 83: 3-12.

Lenka S.K., Katiyar A., Chinnusamy V., Bansal K.C. (2011) Comparative analysis of drought-responsive transcriptome 
in indica rice genotypes with contrasting drought tolerance. Plant Biotech. J. 9: 315-327.

Lescot M., Déhais P., Thijs G., Marchal K., Moreau Y., Van de Peer Y., Rouzé P., Rombauts S. (2002) PlantCARE, a database of plant cis-acting regulatory elements and a portal to tools for in silico analysis of promoter sequences. Nucl. Acids Res. 30(1): 325-327.

Lu Z., Liu D., Liu S. (2007) Two rice cytosolic ascorbate peroxidases differentially improve salt tolerance in transgenic Arabidopsis. Plant Cell Rep. 26: 1909-1917.

Mishra N.S., Tuteja R., Tuteja N. (2006) Signaling through MAP kinase networks in plants. Arch. Environ. Contam. 452: 55-68.

Moumeni A., Satoh K., Kondoh H. et al. (2011) Comparative analysis of root transcriptome profiles of two pairs of drought-tolerant and susceptible rice near-isogenic lines under different drought stress. BMC Plant Biol. 11: 174.

Nouri M.Z., Komatsu S. (2013) Subcellular protein over expression to develop abiotic stress tolerant plants. Fron. Plant Sci. 4: 2.

Pandey S. (2007) Economic costs of drought and rice farmers coping mechanisms. Int. RiceRes. Notes 32(1): 5-11.

Prakash G., Kumar S., Mikawlrawng K. (2014) Metabolic Pathways Engineering: an emerging approach in abiotic stress tolerance in plants. J. Pharmacogn. Phytochem. 3(1): 104-107.

Price A.H., Cairns, J.E., Horton P., Jones H.G., Griffiths H. (2002) Linking drought-resistance mechanisms to drought avoidance in upland rice using a QTL approach: progress and new opportunities to integrate stomatal and mesophyll responses. J. Exp. Bot. 53: 989-1004.

Qian D., Tian L., Qu L. (2015) Proteomic analysis of endoplasmic reticulum stress responses in rice seeds. Sci. Rep. 5: 14255.

Rai V., Singh N.K. (2011) Differential expression for salt and drought stress. Gene series 21651 (GSE21651). Public on Dec 31.

Rao K.P., Richa T., Kumar K., Raghuram B., Sinha A.K. (2010) In silico analysis reveals 75 members of mitogenactivated protein kinase kinase kinase gene family inrice. DNA Res. 17: 139-153.

Ray S., Dansana P.K., Giri J., Deveshwar P., Arora R., Agarwal P., Khurana J.P., Kapoor S., Tyagi A.K. (2011) Modulation of transcription factor and metabolic pathway genes in response to water-deficit stress in rice. Funct. Integr. Genomics 11(1): 157-178.

Roja V. (2014) Molecular mapping of QTL governing water use efficiency in rice. $\mathrm{Ph} . \mathrm{D}$ thesis. Institute of Biotechnology, Acharya NG Ranga Agricultural University, Rajendranagar, Hyderabad-500030, AP, India.

Roja V., Patil S., Deborah D.A. et al. (2016) Finding genomic regions and candidate genes governing water use efficiency in rice. Biol. Plant 60: 757.

Roxas V.P., Lodhi S.A., Garrett D.K., Mahan J.R., Allen R.D. (2000) Stress tolerance in transgenic tobacco seedlings that overexpress glutathione S-transferase/glutathione peroxidase. Plant Cell Physiol. 41: 1229-1234.

Sarris A. (2004) Proceedings of FAO rice conference 2004. FAO, UN.

Shannon P., Markiel A., Ozier O. et al. (2003) Cytoscape: a software environment for integrated models of biomolecular interaction networks. Genome Res. 13(11): 2498-2504.

Sreenivasulu N., Sopory S.K., Kavi Kishor P.B. (2007) Deciphering the regulatory mechanisms of abiotic stress tolerance in plants by genomic approaches. Gene 388: 1-13.

Szklarczyk D., Morris J.H., Cook H., Kuhn M., Wyder S., Simonovic M., Santos A., Doncheva N.T., Roth A., Bork P., Jensen L.J., von Mering C. (2017) The STRING database in 2017: quality-controlled protein-protein association networks, made broadly accessible. Nucl. Acids Res. 45: D362-368.

Tuteja R., Tuteja N. (2013) Analysis of DNA repair helicase $U_{v r D}$ from Arabidopsis thaliana and Oryza sativa. Plant Physiol. Biochem. 71: 254-260.

Van Breusegem F., Vranová E., Dat J.F., Inze D. (2001) The role of active oxygen species in plant signal transduction. Plant Sci. 161: 405-414.

Wang F., Jing W., Zhang W. (2014) The mitogen-activated protein kinase cascade MKK1-MPK4 mediates salt signaling in rice. Plant Sci. 227: 181-189.

Wang J., Qi M., Liu J., Zhang Y. (2015) CARMO: a comprehensive annotation platform for functional exploration of rice multi-omics data. Plant J. 83(2): 359-374.

Wimalasekera R., Scherer G.F.E. (2018) Involvement of mitogen-activated protein kinases in abiotic stress responses in plants. [in:] Plant metabolites and regulation under environmental stress. Ed. Ahmad P., Ahanger M. A., Singh V. P., Tripathi D. K., Alam P., Alyemeni M. N., Academic Press: 389-395.

Yamamoto E., Yonemaru J., Yamamoto T., Yano M. (2012) OGRO: The Overview of functionally characterized Genes in Rice online database. Rice 5: 1-10.

Zhang Z., Zhang Q., Wu J., Zheng X., Zheng S., Sun X. et al. (2013) Gene knockout study reveals that cytosolic ascorbate peroxidase $2(\mathrm{Os} A P X 2)$ plays a critical role in growth and reproduction in rice under drought, salt and cold stresses. PLoS One 8(2): e57472.

Xiang Y., Sun X., Gao S., Qin F., Dai M. (2017) Deletion of an endoplasmic reticulum stress response element in a $\mathrm{ZmPP2C}-\mathrm{A}$ gene facilitates drought tolerance of maize seedlings. Mol. Plant. 10(3): 456-469.

Zimmermann P., Hirsch-Hoffmann M., Hennig L., Gruissem W. (2004) GENEVESTIGATOR. Arabidopsis microarray database and analysis toolbox. Plant Physiol. 136: 2621-2632.

Zinati Z. (2017) Identification of novel genes potentially involved in rice (Oryza sativa L.) drought tolerance. BioTechnologia 98(3): 195-208. 